\title{
Dirac and reduced quantization: A Lagrangian approach and application to coset spaces
}

\author{
C. R. Ordóñez \\ Theory Group, Department of Physics, University of Texas, Austin, Texas 78712 \\ and Department of Physics and Astronomy, Box 1807 Station B, Vanderbilt University, \\ Nashville, Tennessee 37235 \\ J. M. Pons \\ Center for Relativity, Department of Physics, University of Texas, Austin, Texas 78712
}

(Received 1 September 1994; accepted for publication 11 October 1994)

\begin{abstract}
A Lagrangian treatment of the quantization of first class Hamiltonian systems with constraints and Hamiltonian linear and quadratic in the momenta, respectively, is performed. The "first reduce and then quantize" and the "first quantize and then reduce" (Dirac's) methods are compared. A source of ambiguities in this latter approach is pointed out and its relevance on issues concerning self-consistency and equivalence with the "first reduce" method is emphasized. One of the main results is the relation between the propagator obtained $\dot{a}$ la Dirac and the propagator in the full space. As an application of the formalism developed, quantization on coset spaces of compact Lie groups is presented. In this case it is shown that a natural selection of a Dirac quantization allows for full self-consistency and equivalence. Finally, the specific case of the propagator on a two-dimensional sphere $S^{2}$ viewed as the coset space $\mathrm{SU}(2) / U(1)$ is worked out. (O) 1995 American Institute of Physics.
\end{abstract}

\section{INTRODUCTION}

The quantization of constrained systems is plagued with many ambiguities and difficulties which are added to those encountered when dealing with regular systems (ordering problems, Groenwald-van Hove obstruction, ${ }^{1}$ etc.). In particular, in the framework of Dirac quantization, ${ }^{2}$ the preservation of the first class nature of the Hamiltonian and the constraints at the operator level is a highly nontrivial issue. Another important aspect is that of the equivalence between the two standard procedures: (a) Dirac's method of quantizing the "entire" system (i.e., including gauge variables) and obtaining physical states as those annihilated by the operator version of the constraints, and (b) first reducing the classical variables by solving the constraints, and then quantizing as a regular system. These procedures are usually referred to as "quantize first and then reduce" and "reduce first and then quantize," respectively. There seems to be no a priori general principle which guarantees that they agree with each other, let alone one which selects one procedure over the other from the physical point of view. ${ }^{3}$

Kuchar, in a beautiful series of articles, ${ }^{4}$ has discussed these problems in the case of quadratic Hamiltonians and linear constraints. (Kuchař and Hajičček have also studied the case of parametrized theories with quadratic constraints. ${ }^{5}$ ) He finds that, in order to achieve equivalence, one is forced to give up, in the general case, the full Hilbert space structure of the entire system. Following Kuchar's work, McMullan et al. ${ }^{6}$ used the Becchi-Rouet-Stora-Tyutin (BRST) approach of Batalin, Fradkin, and Vilkovisky ${ }^{7}$ to address the same problems. Concurrently, several other groups ${ }^{8}$ have also investigated a wide variety of aspects which arise in the study of the equivalence of these two methods.

The treatment of these problems has been done mostly with canonical (operator) quantization. However, similar issues like the relation between reduced and covariant path integrals (e.g., Faddeev-Popov) also appear. We have investigated these issues in a series of publications ${ }^{9-11}$ and have proven equivalence in this case (see also Ref. 12). 
The work of the groups mentioned above has been mostly done within the Hamiltonian framework. We have chosen to start with a Lagrangian framework (Sec. II). Beyond its wellknown advantages this formalism enables us to encode in a single function all the features of the constrained system. We then proceed with the Hamiltonian formulation (Sec. III), and immediately afterwards we present the two types of quantizations mentioned above (Secs. IV and V). This is followed (Sec. VI) by a discussion of the subtleties and ambiguities present in the "quantize first" approach. Here we also make some remarks on the relevance of the gauge group in this kind of question. In Sec. VII we apply our general setting to the case of quantization on coset spaces of compact Lie groups and then go on to the special case of $S^{2} \approx \mathrm{SU}(2) / \mathrm{U}(1)$ (Sec. VIII). Finally, we present conclusions and outlook in Sec. IX.

\section{THE LAGRANGIAN SETTING}

We are interested in a model with only first class constraints (in phase space, $T^{*} Q$, where $Q$ is the configuration space manifold), all of which should appear as primary constraints in order for them to generate independent gauge transformations. This means that also the Hamiltonian has to be first class, i.e., its Poisson brackets with all the constraints have to vanish on the constraint surface. There is an easy way to cast this information in the Lagrangian formalism. In this article we will consider Lagrangians of the form

$$
L=\frac{1}{2} G_{A B} \dot{Q}^{A} \dot{Q}^{B}-V
$$

where $G_{A B}$ and $V$ are functions of configuration space variables $Q^{A}, A=1, \ldots, N . G_{A B}$ is a singular metric tensor of rank $n<N$. Here we consider explicitly a quantum mechanical system. The extension to field theory is immediate once DeWitt's condensed notation is adopted. ${ }^{13}$ This type of Lagrangian is obtained in Yang-Mills theories when one integrates out $A_{0}$.

The standard construction of the first generation of velocity space $(T Q)$ constraints is as follows. The equation of motion derived from Eq. (2.1) is

$$
G_{A B} \ddot{Q}^{B}=\alpha_{A},
$$

where

$$
\alpha_{A}=\frac{1}{2} G_{B C, A} \dot{Q}^{B} \dot{Q}^{C}-G_{A C, B} \dot{Q}^{B} \dot{Q}^{C}-V_{, A}
$$

If $U^{A}(Q)$ is a null vector of $G_{A B}$, i.e., $G_{A B} U^{B}=0$ identically, then the constraint surface corresponding to the first step of the stabilization algorithm is obtained by requiring

$$
U^{A} \alpha_{A} \approx 0
$$

for every null vector of the metric. We use the weak equality in (2.4) in the Dirac sense. ${ }^{2}$

The work in Ref. 14 shows that in order for the Hamiltonian constraints to be primary first class only, one must require that Eq. (2.4) holds identically, i.e., its solution has to be the entire velocity space. This leads to

$$
U^{C} \Gamma_{C A B}=0
$$

and

$$
U^{C} V_{, C}=0
$$

where

$$
\Gamma_{C A B}=-G_{A B, C}+G_{C B, A}+G_{C A, B}
$$


Using $G_{A B} U^{B}=0$, Eq. (2.5) becomes

$$
U^{C} G_{A B, C}+G_{A C} U_{, B}^{C}+G_{C B} U_{, A}^{C} \equiv\left(\mathscr{L}_{\hat{U}} G\right)_{A B}=0
$$

with

$$
\hat{U}=U^{A}(Q) \frac{\partial}{\partial Q^{A}}
$$

In Eq. (2.8) we have used the standard notation for the Lie derivative.

Hence, the following requirements result:

(a) every null vector (considered as a vector field in configuration space) of the degenerate metric tensor $G$, also has to be a Killing vector for it, and

(b) every such null vector has to be tangent to the equipotential surfaces of the potential $V$.

Notice that the set of null vectors is closed under the Lie bracket. Indeed, let $\hat{U}$ and $\hat{V}$ be two such vectors, i.e.,

$$
i_{\hat{U}} G=i_{\hat{V}} G=0,
$$

where use was made of the standard notation for the contraction of a vector field with a form.

As we saw, they also have to be Killing vectors. Hence, using Eq. (2.8) the following equalities hold:

$$
0=\mathscr{L}_{\hat{U}}\left(i_{\hat{V}} G\right)=i_{\left(\mathscr{L}_{\hat{U}} \hat{V}\right)} G=i_{[\hat{U}, \hat{V}]} G
$$

Therefore, $[\hat{U}, \hat{V}]$ is a null vector.

If we consider a basis for the space of null vectors, $\left\{\hat{U}_{\alpha}, \alpha=1, \ldots, k:=N-n\right\}$, this closure property is written

$$
\left[\hat{U}_{\alpha}, \hat{U}_{\beta}\right]=C_{\alpha \beta}^{\gamma}(Q) \hat{U}_{\gamma} .
$$

As we will see later this relation prefigures the first class character of the constraints that appear in the Hamiltonian formulation.

\section{HAMILTONIAN SETTING}

The usual definition of momenta gives

$$
P_{A}=G_{A B} \dot{Q}^{B},
$$

which implies the following set of primary constraints:

$$
\varphi_{\alpha} \equiv U_{\alpha}^{A} P_{A} \approx 0, \quad \alpha=1, \ldots, k .
$$

Since the rank of the metric $G$ is $n$, Eq. (3.2) is the full set of primary constraints. Equation (2.12) shows that they are first class under the Poisson bracket

$$
\left\{\varphi_{\alpha}, \varphi_{\beta}\right\}=-C_{\alpha \beta}^{\gamma} \varphi_{\gamma} .
$$

As mentioned earlier, these constraints generate gauge transformations through Poisson brackets. Hence the vector fields $\hat{U}_{\alpha}$ generate symmetry transformations in configuration space. This means that configuration space $Q$ is divided into orbits under the action of these vector fields, and that the physical configuration space $\mathscr{A}$ is the quotient of the original one by the orbits. 
Let us now set up the dynamics on $T^{*} Q$. In order for the Lagrangian energy function to be the pullback of the Hamiltonian, which obviously has to be quadratic in the momenta,

$$
H=\frac{1}{2} M^{A B}(Q) P_{A} P_{B}+V,
$$

$M^{A B}$ has to satisfy ( $M$ being symmetric)

$$
M^{A B} G_{A C} G_{B D}=G_{C D} .
$$

Equation (3.5) shows an ambiguity typical of treatments of constrained systems. Indeed, any transformation of the form

$$
M^{A B} \rightarrow M^{\prime A B}=M^{A B}+\lambda^{A \alpha} U_{\alpha}^{B}+\lambda^{B \alpha} U_{\alpha}^{A},
$$

with arbitrary $\lambda^{A \alpha}$ will define another Hamiltonian which will have the same energy function in velocity space. Hence, a first class Hamiltonian system of the type we are considering does not endow configuration space with a unique nonsingular metric structure. This freedom in the choice of a metric structure in configuration space will play an important role in our quantization program.

Finally, it can be shown that given a Hamiltonian of the form (3.4), and a set of first class constraints (3.2), there always exists a Lagrangian of the form (2.1) which gives rise to the Hamiltonian and the constraints. For details see Appendix.

\section{REDUCE FIRST AND THEN QUANTIZE}

We may proceed from either the Hamiltonian or the Lagrangian formulation. As we will show, one obtains the same results. Let us then begin with the Hamiltonian version. There is a natural way of endowing $\mathscr{H}$ with a nonsingular metric structure. Consider the following contravariant tensor field associated to the kinetic term in the Hamiltonian:

$$
M^{A B} \frac{\partial}{\partial Q^{A}} \otimes \frac{\partial}{\partial Q^{B}}
$$

The projection

$$
\pi: Q \rightarrow \mathscr{H}
$$

allows us to assign to Eq. (4.1) a contravariant vector field in $\mathscr{H}$, as long as the following "projectability" condition is fulfilled:

$$
\hat{U}_{\alpha}\left(M^{A B} \frac{\partial f}{\partial Q^{A}} \frac{\partial g}{\partial Q^{B}}\right)=0, \quad \alpha=1, \ldots, k
$$

for any functions $f, g$ of configuration variables $Q^{A}$ such that

$$
\hat{U}_{\alpha} f=\hat{U}_{\alpha} g=0, \quad \alpha=1, \ldots, k
$$

This condition can be recast as

$$
\left(\mathscr{L}_{\hat{U}_{\alpha}} M\right)^{A B} G_{A C} G_{B D}=0,
$$

which is a trivial consequence of Eq. (3.5). Hence we have to our avail this contravariant tensor field in $\mathscr{H}$. 
In order to gain further insight into the structure of this tensor field, we now introduce coordinates $q^{a}, a=1, \ldots, n$, on $\mathscr{A}$. Then the projection $\pi$ is described by the functions $q^{a}(Q)$ that satisfy $\hat{U}_{\alpha} q^{a}=0 ; \alpha=1, \ldots, k, a=1, \ldots, n$.

In these coordinates the components of the new tensor field in $\mathscr{H}$ are

$$
\tilde{g}^{a b}=M^{A B} \frac{\partial q^{a}}{\partial Q^{A}} \frac{\partial q^{b}}{\partial Q^{B}}
$$

The projectability condition shows that $\tilde{g}^{a b}$ only depends on the physical coordinates $q^{a}$.

Notice that $\tilde{g}^{a b}$ so defined is invariant under changes of $M$ of the type (3.6). Moreover, these are the only changes that leave $\tilde{g}^{a b}$ invariant. This result has the direct physical consequence that, for our type of systems, the ambiguities present in the Hamiltonian framework play no role in the reduced first approach to quantization. Hence the physical phase space $T^{*} \mathscr{H}$ is naturally endowed with a Hamiltonian

$$
h=\frac{1}{2} \tilde{g}^{a b} p_{a} p_{b}+V
$$

Now we will show that $\tilde{g}$ is nonsingular. In order to prove this it is convenient to work with a coordinate system in $Q$ adapted to the orbit structure, we then add, to the physical coordinates $q^{a}$, $a=1, \ldots, n$, which label the orbits, a new set of functions (which parametrize the orbits) $q^{\alpha}$, $\alpha=1, \ldots, k$ such that $\operatorname{det}\left|\hat{U}_{\alpha} \hat{q}^{\beta}\right| \neq 0$. The entire set will henceforth be termed "adapted coordinate system."

In this system the Lagrangian metric $G_{A B}$ and the null vectors are written

$$
G=\left(\begin{array}{ll}
g_{a b} & g_{a \beta} \\
g_{\alpha b} & g_{\alpha \beta}
\end{array}\right)
$$

and

$$
\hat{U}_{\alpha}=U_{\alpha}^{\beta}\left(q^{a}, q^{\gamma}\right) \frac{\partial}{\partial q^{\beta}}
$$

respectively.

The condition that these vectors are null vectors for this metric immediately leads to

$$
g_{\alpha b}=g_{a \beta}=g_{\alpha \beta}=0
$$

and the Killing condition for these vectors becomes

$$
\hat{U}_{\alpha}\left(g_{a b}\right)=0, \quad \alpha=1, \ldots, k .
$$

This last result guarantees that $g_{a b}$ only depends on the physical coordinates $q^{a}, a=1, \ldots, n$. Equations (4.8) and (4.10) display the nonsingularity of $g_{a b}$ because the rank of $G$ is $n$.

Equation (3.5) now becomes

$$
M=\left(\begin{array}{cc}
g^{a b} & m^{a \beta} \\
m^{\alpha b} & m^{\alpha \beta}
\end{array}\right),
$$

where $g^{a b}=\left(g_{a b}\right)^{-1}$, and the remaining components of $M$ are arbitrary.

Finally, the projection $\pi$ to $\mathscr{H}$ of the contravariant tensor (4.1) leads to the identification $\tilde{g}^{a b}=g^{a b}$. This proves that the contravariant metric tensor defined in $\mathscr{A l}$ is nonsingular. The Hamiltonian formulation on $T^{*} \mathscr{O}$ is now completed. 
Let us next proceed with the Lagrangian version. Taking advantage of the form of $G$ in the adapted coordinate system, it is immediate to see that there is a unique function $\ell$ on $T \mathscr{H}$ such that its pullback $\pi^{*} \ell$ under the derivative mapping $\pi^{\prime}$ induced by the projection $\pi$, i.e.,

$$
\pi^{\prime}: T Q \rightarrow T \mathscr{H}
$$

is just the original Lagrangian $L$

$$
\ell=\frac{1}{2} g_{a b} \dot{q}^{a} \dot{q}^{b}-V, \quad \pi^{\prime *} \ell=L .
$$

Summarizing: we have found the Hamiltonian and the Lagrangian for the reduced system. They are obviously related by a Legendre transformation. In the construction of these functions it now becomes quite clear that the metric $g_{a b}$ is the one to be used to define the measure on $\mathscr{A B}$. From this point on, we can proceed within the standard quantization scheme for regular systems. (See Refs. 4 and 15 for details.) Notice that in this geometrical framework no explicit gauge fixing was performed.

The inner product that defines the Hilbert space $\mathscr{H}_{r}=\mathscr{L}^{2}\left(\mathscr{H},|g|^{1 / 2}\right)$ is defined as

$$
\left\langle\psi_{1} \mid \psi_{2}\right\rangle=\int_{,} d^{n} q|g|^{1 / 2} \psi_{1}^{*}(q) \psi_{2}(q)
$$

where $|g|$ is the determinant of $g_{a b}$. The dynamical evolution (Schrödinger equation) will be given by the Hamiltonian operator

$$
\hat{h}=-\frac{1}{2} \Delta_{g}+V
$$

where $\Delta_{g}$ is the Laplace-Beltrami operator

$$
\Delta_{g}=|g|^{-1 / 2} \frac{\partial}{\partial q^{a}}|g|^{1 / 2} g^{a b} \frac{\partial}{\partial q^{b}} .
$$

In Kuchař's words, Eq. (4.16) implements the "principle of minimal coupling"; curvature terms are not included.

The configuration variables $q^{a}$ become multiplicative operators as in the usual case, whereas, in order to retain Hermiticity, the canonical momenta $p_{a}$ are quantized as follows:

$$
p_{a} \rightarrow-i\left(\frac{\partial}{\partial q^{a}}+\frac{1}{2}|g|^{-1 / 2}|g|_{, a}^{1 / 2}\right) .
$$

In the next section we reverse the order of things and proceed to "quantize first and then reduce."

\section{QUANTIZE FIRST AND THEN REDUCE, DIRAC'S METHOD}

As we discussed in Sec. III we always have the freedom to choose a nonsingular metric $M$ with which we can immediately write down an inner product.

$$
\left\langle\psi_{1} \mid \psi_{2}\right\rangle=\int_{Q} d^{N} Q|M|^{1 / 2} \psi_{1}^{*}(Q) \psi_{2}(Q)
$$

This inner product defines the Hilbert space $\mathscr{H}=\mathscr{L}^{2}\left(Q,|M|^{1 / 2}\right)$. [Here $|M|=\operatorname{det} M_{A B}$, $M_{A B}=\left(M^{A B}\right)^{-1}$.] We will assume that the quantum operators associated with the classical constraints and the Hamiltonian still form a first class system with respect to the commutator algebra. For a further discussion of this assumption we refer to the next section. 
The operator assignment for the classical constraints is dictated by the requirement that the physical wave function only depend upon physical variables, i.e., variables describing $\mathscr{M}$

$$
\varphi_{\alpha} \rightarrow \hat{\varphi}_{\alpha}:=\hat{U}_{\alpha}=U_{\alpha}^{A} \frac{\partial}{\partial Q^{A}} .
$$

Notice that Eq. (2.12) guarantees that the classical constraints $\varphi_{\alpha}$ are realized as a set of first class quantum operators. The Hamiltonian in $\mathscr{H}$ is chosen as in Sec. IV, i.e.,

$$
\hat{H}=-\frac{1}{2} \Delta_{M}+V,
$$

where

$$
\Delta_{M}=|M|^{-1 / 2} \frac{\partial}{\partial Q^{A}}|M|^{1 / 2} M^{A B} \frac{\partial}{\partial Q^{B}}
$$

and it is assumed to satisfy the first class condition

$$
\left[\hat{U}_{\alpha}, \hat{H}\right]=\lambda_{\alpha}^{\beta}(Q) \hat{U}_{\beta} .
$$

The physical Hilbert space $\mathscr{H}_{p}$ in this framework is then obtained as the subspace of $\mathscr{H}$ defined by those states which satisfy

$$
\left.\hat{U}_{\alpha} \mid \text { Phys }\right\rangle=0, \quad \alpha=1, \ldots, k
$$

In wave function language

$$
\hat{U}_{\alpha}\langle Q \mid \psi\rangle \equiv \hat{U}_{\alpha} \psi(Q)=0
$$

This is equivalent to

$$
\psi(Q)=\tilde{\psi}\left(f^{a}(Q)\right)
$$

where $q^{a}=f^{a}(Q)$ defines explicitly the projection $\pi$, Eq. (4.2). Physical position kets $\left|q^{a}\right\rangle_{\mathrm{Ph}}$ are those states in $\mathscr{H}$ defined by

$$
\mathrm{Ph}\left\langle q^{a} \mid \psi\right\rangle=\tilde{\psi}\left(q^{a}\right)
$$

with $|\psi\rangle$ satisfying Eq. (5.6). It is convenient to expand these states in terms of the position states $|Q\rangle$ in $\mathscr{H}$. Consider the following identities, valid for arbitrary $\tilde{\psi}$ :

$$
\tilde{\psi}\left(q^{a}\right)={ }_{\mathrm{Ph}}\left\langle q^{a} \mid \psi\right\rangle=\int d^{N} Q|M|^{1 / 2}{ }_{\mathrm{Ph}}\left\langle q^{a} \mid Q\right\rangle\langle Q \mid \psi\rangle=\int d^{N} Q|M|^{1 / 2}{ }_{\mathrm{Ph}}\left\langle q^{a} \mid Q\right\rangle \tilde{\psi}\left(f^{a}(Q)\right),
$$

which implies that

$$
\operatorname{Ph}\left\langle q^{a} \mid Q\right\rangle=\frac{1}{\mu\left(q^{a}\right)} \delta^{n}\left(q^{a}-f^{a}(Q)\right)
$$

where

$$
\mu\left(q^{a}\right)=\int d^{N} Q|M|^{1 / 2} \delta^{n}\left(q^{a}-f^{a}(Q)\right)
$$


Notice that Eq. (5.11) requires that these volumes be finite at every point $q^{a}$. This is equivalent to requiring that the physical wave functions $\psi$ be normalizable in $\mathscr{H}$, since they are constant along each orbit. Hence, in a rigorous sense, the "first quantize" approach is only applicable in the cases where this condition is satisfied. Of course, one can be more practical, and carry out the Dirac construction in a more formal fashion ignoring details of normalization in $\mathscr{H}$. In our article we are going to assume that $\mu\left(q^{a}\right)$ is finite at every $q^{a}$.

It will prove convenient to rewrite $\mu\left(q^{a}\right)$ without the delta function. For this purpose, lct us use the adapted coordinate system $\left(q^{a}, q^{\alpha}\right)$. Consider the following identity:

$$
1=\int d^{k} q \delta^{k}\left(q^{\alpha}-f^{\alpha}(Q)\right)
$$

where the functions $f^{\alpha}$ give the explicit realization of the coordinate change. Inserting Eq. (5.13) into Eq. (5.12) we obtain

$$
\begin{aligned}
\mu\left(q^{a}\right) & =\int d^{k} q \int d^{N} Q|M|^{1 / 2} \delta^{n}\left(q^{a}-f^{a}(Q)\right) \delta^{k}\left(q^{\alpha}-f^{\alpha}(Q)\right) \\
& =\int d^{k} q \int d^{N} Q|M|^{1 / 2} \delta^{N}(q-f(Q)) \\
& =\int d^{k} q \int d^{N} Q|M|^{1 / 2} \frac{1}{|\partial q / \partial Q|} \delta^{N}(Q-Q(q)) \\
& =\int d^{k} q|M|^{1 / 2} \frac{1}{|\partial q / \partial Q|}=\int d^{k} q|m|^{1 / 2},
\end{aligned}
$$

where $|m|$ is the determinant of the metric in the adapted coordinate system.

In Ref. 9 we proved the following important factorization property of $m$, in adapted coordinates:

$$
|m|=\left|g_{a b}\right|\left|m_{\alpha \beta}\right|=:|g|\left|m_{\alpha \beta}\right|,
$$

where $g_{a b}\left(q^{a}\right)$ is the "physical" metric of Sec. IV. This implies that Eq. (5.14) can also be written as

$$
\mu\left(q^{a}\right)=\int d^{k} q|g|^{1 / 2}\left|m_{\alpha \beta}\right|^{1 / 2}=|g|^{1 / 2} \int d^{k} q\left|m_{\alpha \beta}\right|^{1 / 2}=:|g|^{1 / 2} \mathscr{V}\left(q^{a}\right)
$$

$7\left(q^{a}\right)$ is naturally identified as the volume of the orbit labeled by $q^{a}$ (recall the $q^{\alpha}$ s are "gauge" variables). Notice that since by hypothesis $\mu\left(q^{a}\right)$ is finite, so is $\mathscr{T}\left(q^{a}\right)$ [or, as this formula shows, one can demand finiteness of $\mathscr{Z}\left(q^{a}\right)$ instead from the outset].

We can identify $\mu\left(q^{a}\right)$ as the measure on $\mathscr{H}$ as follows: compute the inner product of two physical states $|\psi\rangle,|\chi\rangle$ in $\mathscr{H}_{p}$ 


$$
\begin{aligned}
\langle\psi \mid \chi\rangle=\int d^{N} Q|M|^{1 / 2} \psi^{*}(Q) \chi(Q) & =\int d^{N} Q|M|^{1 / 2} \tilde{\psi}^{*}\left(f^{a}(Q)\right) \tilde{\chi}\left(f^{a}(Q)\right) \\
& =\int d^{n} q d^{k} q\left|\frac{\partial Q}{\partial q}\right||M|^{1 / 2} \tilde{\psi}^{*}\left(q^{a}\right) \tilde{\chi}\left(q^{a}\right) \\
& =\int d^{n} q\left[\int d^{k} q|m|^{1 / 2}\right] \tilde{\psi}^{*}\left(q^{a}\right) \tilde{\chi}\left(q^{a}\right) \\
& =\int d^{n} q \mu\left(q^{a}\right) \tilde{\psi}^{*}\left(q^{a}\right) \tilde{\chi}\left(q^{a}\right) .
\end{aligned}
$$

In particular, one can show that

$$
{ }_{\mathrm{Ph}}\left\langle q_{1}^{a} \mid q_{2}^{a}\right\rangle_{\mathrm{Ph}}=\frac{1}{\mu\left(q_{1}^{a}\right)} \delta^{n}\left(q_{1}^{a}-q_{2}^{a}\right) .
$$

The physical position states $\left|q^{a}\right\rangle_{\mathrm{Ph}}$ can be written in terms of the position states $|Q\rangle$ in $\mathscr{H}$. Indeed, using Eq. (5.11) we obtain

$$
\left|q^{a}\right\rangle_{\mathrm{Ph}}=\frac{1}{\mu\left(q^{a}\right)} \int d^{N} Q|M|^{1 / 2} \delta^{n}\left(q^{a}-f^{a}(Q)\right)|Q\rangle
$$

In adapted coordinates, using the factorization property, Eq. (5.15), we obtain

$$
\left|q^{a}\right\rangle_{\mathrm{Ph}}=\frac{1}{\mathscr{V}\left(q^{a}\right)} \int d q^{k}\left|m_{\alpha \beta}\right|^{1 / 2}\left|q^{a}, q^{\alpha}\right\rangle
$$

This formula displays the nature of $\left|q^{a}\right\rangle_{\mathrm{Ph}}$ as a kind of average over the gauge degrees of freedom of $\left|q^{a}, q^{\alpha}\right\rangle$, and this connection will be useful when we compute the propagator in $\mathscr{H}_{p}$. The projection onto $\mathscr{H}_{p}$ can readily be written as

$$
\mathscr{P}=\int d^{n} q \mu\left(q^{a}\right)\left|q^{a}\right\rangle_{\mathrm{PhPh}}\left\langle q^{a}\right|
$$

This operator can also be expressed in terms of the states $|Q\rangle$

$$
\mathscr{P}=\int d^{N} Q d^{N} Q^{\prime}|M|^{1 / 2}\left|M^{\prime}\right|^{1 / 2} \frac{1}{\mu\left(f^{n}(Q)\right)} \delta^{n}\left(f^{a}(Q)-f^{a}\left(Q^{\prime}\right)\right)|Q\rangle\left\langle Q^{\prime}\right|
$$

As expected, $\not{P}$ projects states $|Q\rangle$ into $\left|q^{a}\right\rangle_{\mathrm{Ph}}$

$$
\mathscr{P} Q\rangle=\left|q^{a}\right\rangle_{\mathrm{Ph}}
$$

where $q^{a}=f^{a}(Q)$. Finally, we are now in the position of being able to relate the propagator in $\not \mathscr{C}$ with the propagator in $\mathscr{H}_{p}$. Since we have a well-defined Hamiltonian $\hat{H}$ in $\mathscr{H}$ which, due to the first class nature-even at the quantum level-of our system, evolves physical states into physical states, then we can immediately write down the extension of formula (5.20) to the Heisenberg representation

$$
\left|q^{a}, t\right\rangle_{\mathrm{Ph}}=\frac{1}{\mathscr{Y}\left(q^{a}\right)} \int d q^{k}\left|m_{\alpha \beta}\right|^{1 / 2} \mid\left(q^{a}, q^{\alpha}, t\right\rangle
$$




$$
\operatorname{Ph}\left\langle q_{2}^{a}, t_{2} \mid q_{1}^{a}, t_{1}\right\rangle_{\mathrm{Ph}}=\frac{1}{\mathscr{W}\left(q_{2}^{a}\right) \mathscr{T}\left(q_{1}^{a}\right)} \int d q_{2}^{k} d q_{1}^{k}\left|m_{\alpha \beta}\left(q_{2}\right)\right|^{1 / 2}\left|m_{\alpha \beta}\left(q_{1}\right)\right|^{1 / 2}\left\langle q_{2}^{a}, q_{2}^{\alpha}, t_{2} \mid q_{1}^{a}, q_{1}^{\alpha}, t_{1}\right\rangle
$$

Equation (5.25) is one of the main results of this article.

\section{QUANTUM FIRST CLASS CONDITION AND THE GAUGE GROUP}

In this section we want to explore the possibilities afforded by virtue of the freedom in the choice of a nonsingular $M^{A B}$ satisfying Eq. (3.5). Its selection should be constrained by at least the requirement that the first class nature of the system be preserved at the quantum level. This is essential for the implementation of the Dirac program. Once this has been achieved, we can then address the issue of coincidence of Dirac's method with the "first reduce" quantization scheme. (4.12),

Quantum first class condition. The expression for $M^{A B}$ in the adapted coordinate system, Eq.

$$
M=\left(m^{A^{\prime} B^{\prime}}\right)=\left(\begin{array}{cc}
g^{a b} & m^{a \beta} \\
m^{\alpha b} & m^{\alpha \beta}
\end{array}\right)
$$

allows us to write the Laplace-Beltrami operator (acting on scalars) as

$$
\Delta_{M}=m^{A^{\prime} B^{\prime}} \nabla_{A^{\prime}} \partial_{B^{\prime}}=m^{A^{\prime} B^{\prime}}\left(\partial_{A^{\prime}} \partial_{B^{\prime}}-\Gamma_{A^{\prime}, B^{\prime}}^{C^{\prime}} \partial_{C^{\prime}}\right)
$$

or, on physical wave functions

$$
\left.\Delta_{M}\right|_{\mathrm{Ph}}=g^{a b} \partial_{a} \partial_{b}-m^{A^{\prime} B^{\prime}} \Gamma_{A^{\prime} B^{\prime}}^{a} \partial_{a} .
$$

The first class condition at the quantum level, Eq. (5.5), is equivalent to preserving the physical nature of the states under Hamiltonian evolution, which in our case, in virtue of the gauge invariance of the potential, Eq. (2.6), reads

$$
\left.\hat{U}_{\alpha} \Delta_{M} \mid \text { Phys }\right\rangle=0, \quad \alpha=1, \ldots, k,
$$

which leads, considering the form of Eq. (6.2), to the following sufficient and necessary condition:

$$
\hat{U}_{\alpha}\left(m^{A^{\prime} B^{\prime}} \Gamma_{A^{\prime} B^{\prime}}^{a}\right)=0, \quad \alpha=1, \ldots, k, \quad a=1, \ldots, n .
$$

In other words, $m^{A^{\prime} B^{\prime}} \Gamma_{A^{\prime} B^{\prime}}^{a}$ has to be a gauge invariant object.

One criterion for the determination of $m^{A^{\prime} B^{\prime}}$ in order to fulfill Eq. (6.4) is to demand that the generators $\hat{U}_{\alpha}, \alpha=1, \ldots, k$ of the gauge transformations be Killing vectors for the metric (this condition is taken as the starting point in the usual treatments). This guarantees that the Hamiltonian $\hat{H}$ of Eq. (5.3) commutes with these Killing vectors. For this to be achieved, these generators have to form a Lie algebra. Hitherto we have not had to mention the freedom to choose a basis of the space of null vectors of the Lagrangian metric $G_{A B}$. This corresponds to the invariance of the constraint surface defined by Eq. (3.2) under rescaling of the primary Hamiltonian constraints (this invariance plays a central role in Kuchar's work ${ }^{4}$ ). By selecting the generators $\hat{U}_{\alpha}, \alpha=1, \ldots, k$ to form a Lie algebra (i.e., constant structure coefficients), we would be spoiling this rescaling invariance. Moreover, different ways of selecting the generators may lead to different Lie algebra structures, and hence different Killing metrics. This will likely lead to different quantizations. These reflections prompt us to discuss the nature of the gauge group at more length. 
The gauge group. At a fixed time, instead of considering the gauge group acting on the space of trajectories, we can consider its action on $Q$. Here the gauge group is defined as the group of transformations (diffeomorphisms) that leave the orbits invariant. In adapted local coordinates

$$
q^{a}, q^{\alpha} \rightarrow \bar{q}^{a}, \bar{q}^{\alpha}, \quad \text { with } \quad \tilde{q}^{a}=q^{a}, \quad \bar{q}^{\alpha}=\bar{q}^{\alpha}\left(q^{a}, q^{\alpha}\right) .
$$

The null vector fields of the Lagrangian metric, described by the basis $\hat{U}_{\alpha}, \alpha=1, \ldots, k$, are tangent to the orbits and generate uniparametric subgroups of the gauge group. There is a great deal of freedom to choose these generators. In particular it may be possible to choose $k$ independent generators spanning a Lie algebra structure which will be associated with a $k$-dimensional Lie subgroup. Locally, one can always obtain these Lie subgroups of the gauge group, but global obstructions may rule out some of them. One simple example of such a local construction is furnished by the holonomic basis $\partial / \partial q^{\alpha}, \alpha=1, \ldots, k$, which provides us with an Abelian subgroup. These considerations serve to illustrate the following important point: any of these subgroups can be used to reconstruct the orbits, and hence, the full gauge group. So, in this geometrical picture, there is no loss of information if we "cut down" the gauge group to one of these subgroups. ${ }^{16}$

If we assume the Killing condition one can try to make contact with the "reduce first" method as follows. First of all, as we saw in Sec. V, we have to check for consistency of the dynamical evolution in both approaches, i.e.,

$$
\left.\Delta_{M}\right|_{\mathrm{Ph}}=\Delta_{g}
$$

where $\Delta_{g}$ is defined by Eq. (4.17). In the adapted coordinate system, Eq. (6.6) implies

$$
m^{A^{\prime} B^{\prime}} \Gamma_{A^{\prime} B^{\prime}}^{a}=g^{c b} \tilde{\Gamma}_{c b}^{a} .
$$

In Eq. (6.7) $\tilde{\Gamma}_{c b}^{a}$ is computed with the reduced metric. This would be readily guaranteed if the metric $M$ in adapted coordinates reads as follows:

$$
m^{A^{\prime} B^{\prime}}=\left(\begin{array}{cc}
g^{a b}\left(q^{a}\right) & 0 \\
0 & g^{\alpha \beta}\left(q^{\alpha}\right)
\end{array}\right) .
$$

Consistency of this form of the metric and the Killing condition demands that

$$
U_{\alpha, a}^{\beta}=0,
$$

which, considering the freedom in the choice of $\hat{U}_{\alpha}$ to form a Lie algebra does not seem to be, at least locally, too restrictive.

The other issue to study in order to check for matching hetween the two approaches is the relation between the measures in both cases. At this point the factorization property, Eq. (5.15), is very useful. Notice that in general the volume of the orbit $\mathscr{T}\left(q^{a}\right)$ is a function of $q^{a}$. In the case where the metric takes the form (6.8) a $q^{a}$ dependence would arise if the range of integration in Eq. (5.16) were to depend on the orbit which is a global problem that one should not discard $a$ priori. Equation (5.16) shows that in the general case, when $\not /\left(q^{a}\right)$ is not a constant, there is a good chance for the two procedures not to agree, since the measures are not equal. One could attempt to remedy this deficiency by absorbing $\mathscr{T}\left(q^{a}\right)$ in a redefinition of the wave functions. Nevertheless, this will generally spoil the equivalence between the dynamics which had been previously established. In the situation where $\mathscr{V}$ is a constant, one would be tempted to conclude that equivalence has been achieved. But there might be some issues concerning the domain of the operators when the self-adjointness condition is implemented, which we have taken for granted. This clearly deserves further study on a case by case basis.

Despite the many possibilities shown here for quantization $\grave{a}$ la Dirac, there is a wide class of theories where equivalence is rigorously obtained, with a natural selection of the Lie subgroup of 
the gauge group and the metric. This is the case of quantization on coset spaces of compact Lie groups, which is the subject of the next section. Before that it is worth to mention that Kuchar ${ }^{17}$ has shown in a simple model the nonequivalence between Dirac and reduced quantization. His model has a field theoretical application in scalar electrodynamics but, as is pointed out by Kuchar, renormalization problems obscure in this case the issue of the equivalence of both approaches.

\section{APPLICATION: QUANTIZATION ON COSET SPACES}

We will first establish notation and then explain how to set up the constrained system of interest for us on coset spaces.

Our configuration manifold will be a compact Lie group $G$ of dimension $N$. Its Lie algebra will be realized by means of left-invariant vector fields $\hat{U}_{A}, A=1, \ldots, N$

$$
\left[\hat{U}_{A}, \hat{U}_{B}\right]=C_{A B}^{C} \hat{U}_{C}
$$

where the structure constants satisfy, besides the obvious ones, the following antisymmetry property:

$$
C_{A B}^{C}=-C_{A C}^{B}
$$

which can always be achieved for any compact Lie algebra. ${ }^{18}$

On coordinates $Q^{A}, A=1, \ldots, N$ our left-invariant vector fields can be written as $\hat{U}_{A}=U_{A}^{B}(Q) \partial / \partial Q^{B}$, and its dual left-invariant forms as $\Omega^{A}=\Omega_{B}^{A}(Q) d Q^{B}, \Omega_{A}^{B}=\left(U_{A}^{B}\right)^{-1} . G$ is endowed with a nonsingular left-invariant metric $M_{A B}$ which is a Killing metric for the vector fields $\hat{U}_{A}, A=1, \ldots, N$. It has the form

$$
M_{A B}=\Omega_{A}^{C} \Omega_{B}^{D} \delta_{C D}
$$

In order to introduce a constrained system in a natural fashion, we will extract from Eq. (7.3) a singular metric which will define the kinetic term in the Lagrangian for such a system. One way to achieve this is simply to restrict the range of the summation index in Eq. (7.3) to $a=1, \ldots, n$ $<N$. Indeed, $\hat{U}_{\alpha}$, where $\alpha$ runs over the complementary set of indices, form a basis of null vectors for this new metric $G_{A B}=\Omega_{A}^{a} \Omega_{B}^{a}$. At this point, we start making contact with our Lagrangian setting of Sec. II. In this case, condition (2.8) implies that the structure constants have to satisfy

$$
C_{\alpha \beta}^{a}=0
$$

and

$$
C_{b \alpha}^{a}=-C_{a \alpha}^{b} .
$$

Equation (7.4) is the statement that the $\hat{U}_{\alpha}, \alpha=1, \ldots, k=N-n$ form a subalgebra of the original Lie algebra

$$
\left[\hat{U}_{\alpha}, \hat{U}_{\beta}\right]=C_{\alpha \beta}^{\gamma} \hat{U}_{\gamma}
$$

Equation (7.5) does not give new information, since this is already guaranteed by Eq. (7.2).

This subalgebra generates a Lie subgroup $K$ of $G$. The quotient manifold $\mathscr{H}$ of Sec. IV is then the coset space $G / K$. To fix ideas, it is convenient to write our singular Lagrangian

$$
L=\frac{1}{2} G_{A B} \dot{Q}^{A} \dot{Q}^{B}-V,
$$


where $G_{A B}$ was just defined above, and $V$ is a potential function satisfying, according to Eq. (2.6), $U_{\alpha}^{A} V_{, A}=0, \alpha=1, \ldots, k$.

Now we present the Hamiltonian formalism. According to Eq. (3.2) the constraints are

$$
\varphi_{\alpha} \equiv U_{\alpha}^{A}(Q) P_{A} \approx 0, \quad \alpha=1, \ldots, k
$$

Due to the particular structure of our metric $G_{A B}$ it is straightforward to verify that the nonsingular contravariant metric $M^{A B}=U_{C}^{A} U_{C}^{B}$ (which is the inverse of the covariant nonsingular metric $M_{A B}$ that we started with) is a solution of Eq. (3.5). This metric structure provides us with the invariant measure on $G$, and it is such a natural structure on it that we will henceforth always work with it. Therefore, Eq. (3.4) becomes

$$
H=\frac{1}{2} M^{A B} P_{A} P_{B}+V,
$$

with $M^{A B}=U_{C}^{A} U_{C}^{B}$. Now we have all the ingredients to quantize according to the two schemes presented in Secs. IV and V. The "first reduce and then quantize" procedure is readily implemented since all we have to do is use the metric tensor $M$ to obtain $\tilde{g}$ for $G / K$ [Eq. (4.6)]. This assignment defines the Hilbert space structure and the quantum dynamics according to Eqs. (4.15)-(4.18). This completes the "first reduce..." program.

In order to implement the "first quantize and then reduce" scheme, we first have to check the first class character of the quantum Hamiltonian, i.e., Eq. (5.5). The first class nature of the constraints is already assured by Eq. (7.6). In our case the Laplacian operator is

$$
\Delta_{M}=|M|^{-1 / 2} \frac{\partial}{\partial Q^{A}}|M|^{1 / 2} M^{A B} \frac{\partial}{\partial Q^{B}} .
$$

Now, since the $\hat{U}_{A}, A=1, \ldots, N$ are Killing vectors for $M$, they are divergenceless

$$
|M|^{-1 / 2} \frac{\partial}{\partial Q^{A}}|M|^{1 / 2} U_{C}^{A}=0, \quad C=1, \ldots, N,
$$

which allows us to write $\Delta_{M}$ as

$$
\Delta_{M}=\hat{U}_{C} \hat{U}_{C}
$$

Using Eq. (7.12) and the antisymmetry property $C_{A B}^{C}=-C_{A C}^{B}$ is a matter of straightforward algebra to verify that

$$
\left[\Delta_{M}, \hat{U}_{A}\right]=0, \quad A=1, \ldots, N,
$$

which was already guaranteed by the Killing vector condition. Hence, $\hat{H}$ passes the test. This guarantees a consistent quantization in $\mathscr{H}_{p}$, in the sense defined in Sec. V. Furthermore, since the gauge orbits (generated by $K$ ) are compact, all the formulas derived in that section apply in a rigorous sense. To show equivalence we now proceed in two steps: first, we will show that the measure on the physical space $\mathscr{B}_{p}$ coincides, up to an irrelevant constant factor, with the measure obtained from the "reduced first" procedure-this shows that, in fact, the two Hilbert spaces are the same. Then, we will see that the dynamics in both cases are identical.

The measures. In the "first reduce" procedure, the metric in $G$ is defined as

$$
g^{a b}=M^{A B} \frac{\partial q^{a}}{\partial Q^{A}} \frac{\partial q^{b}}{\partial Q^{B}}=U_{D}^{A} U_{D}^{B} \frac{\partial q^{a}}{\partial Q^{A}} \frac{\partial q^{b}}{\partial Q^{B}}=\hat{U}_{D}\left(q^{a}\right) \hat{U}_{D}\left(q^{b}\right)=\hat{U}_{d}\left(q^{a}\right) \hat{U}_{d}\left(q^{b}\right)=U_{d}^{a} U_{d}^{b},
$$


where we used $\hat{U}_{\alpha}\left(q^{a}\right)=0$. The measure is then

$$
\mu_{R}=|g|^{1 / 2}=\left|g^{a b}\right|^{-1 / 2}=\left|U_{a}^{b}\right|^{-1} .
$$

Now consider the "first quantize" approach. In the adapted coordinate system, $Q^{A^{\prime}}=\left(q^{a}, q^{\alpha}\right)$, we showed factorization in general, Eq. (5.15). The only possible obstruction to equivalence is the dependence of $\mathcal{Z}^{\prime}\left(q^{a}\right)$ on $q^{a}$. Let us now compute $\mathscr{T}\left(q^{a}\right)$ in this case. From Eq. (7.3) we get

$$
|m|^{1 / 2}=\left|\hat{U}_{D}\left(Q^{A^{\prime}}\right)\right|^{-1}=\left|U_{a}^{b}\right|^{-1}\left|U_{\alpha}^{\beta}\right|^{-1}=\mu_{R}\left(q^{a}\right)\left|U_{\alpha}^{\beta}\right|^{-1}=\mu_{R}\left(q^{a}\right)\left|\Omega_{\alpha}^{\beta}\right| \equiv \mu_{R}\left(q^{a}\right)|\Omega|
$$

Therefore, the volume of the orbit is

$$
\mathscr{Z}\left(q^{a}\right)=\int d^{k} q|\Omega|
$$

We now prove that Eq. (7.17) is indeed a constant. To see this, it is convenient to rewrite $\mathscr{Z}$ in intrinsic notation. Consider the injection

$$
i_{q^{a}}: O\left(q^{a}\right) \rightarrow G
$$

Then we have

$$
\mathscr{T}\left(q^{a}\right)=\int_{\left(q^{a}\right)} i_{q a}^{*} \Sigma,
$$

where $\Sigma=\Omega^{1} \wedge \Omega^{2} \wedge \ldots \wedge \Omega^{k}$ and the indices $1,2, \ldots, k$ are the $\alpha$-type indices. $i_{q^{\alpha}}^{*} \Sigma$ is the pullback of $\Sigma$ under Eq. (7.18). We should remind ourselves that $\Sigma$ is by construction left-invariant. Now consider an element $g \in G$, but $g \notin K$. It maps orbit into other orbits

$$
g: \mathscr{Q}\left(q^{a}\right) \rightarrow \mathscr{Q}\left(\tilde{q}^{a}\right)
$$

Then the following chain of equalities holds:

$$
\mathscr{Y}\left(\tilde{q}^{a}\right) \equiv \int_{C\left(\tilde{q}^{a}\right)} i_{q^{a}}^{*} \Sigma=\int_{C_{\left(q^{a}\right)}} g^{*}\left(i_{\tilde{q}^{a}}^{*} \Sigma\right)=\int_{\mathscr{C}\left(q^{a}\right)} i_{q^{a}}^{*}\left(g^{*} \Sigma\right)=\int_{\mathscr{G}_{\left(q^{a}\right)}} i_{q^{a}}^{*} \Sigma \equiv \mathscr{T}\left(q^{a}\right) .
$$

The first equality comes from a passive interpretation of the action of $g$ as a change of variables in the same orbit. The second one is just a consequence of the fact that $i_{q a} \circ g=g \circ i_{q a}$. To obtain the last one we used the left-invariance of $\Sigma$. This proves that all orbits have the same volume. Therefore the measures are equal up to a normalization constant.

The dynamics. Now we proceed to show that the Laplacian operator $(7.10), \Delta_{M}$, when restricted to $\mathscr{F}_{p}$ coincides with the Laplacian operator (4.17) $\Delta_{g}$ of the "reduce first" approach. First we observe that Eq. (7.12) allows us to write the restriction of $\Delta_{M}$ on $\mathscr{H}_{p}$ as

$$
\left.\Delta_{M}\right|_{p}=\hat{U}_{a} \hat{U}_{a}
$$

where we have used $\hat{U}_{\alpha} q^{a}=0$. Explicitly, in the adapted coordinate system

$$
\left.\Delta_{M}\right|_{p}=U_{a}^{b} \frac{\partial}{\partial q^{b}} U_{a}^{c} \frac{\partial}{\partial q^{c}}+\left.U_{a}^{\alpha} \frac{\partial}{\partial q^{\alpha}} U_{a}^{c} \frac{\partial}{\partial q^{c}}\right|_{Z_{p}}=U_{a}^{b} \frac{\partial}{\partial q^{b}} U_{a}^{c} \frac{\partial}{\partial q^{c}}+U_{a}^{\alpha} U_{a, \alpha}^{c} \frac{\partial}{\partial q^{c}}
$$

On the other hand 


$$
\Delta_{g}=|g|^{-1 / 2} \partial_{b}|g|^{1 / 2} g^{b c} \frac{\partial}{\partial q^{c}}=U_{a}^{b} \frac{\partial}{\partial q^{b}} U_{a}^{c} \frac{\partial}{\partial q^{c}}+|g|^{-1 / 2}\left(|g|^{1 / 2} U_{a}^{b}\right)_{, b} U_{a}^{c} \frac{\partial}{\partial q^{c}}
$$

Hence

$$
\left.\Delta_{M}\right|_{*_{p}}-\Delta_{g}=\left(U_{a}^{\alpha} U_{a, \alpha}^{c}-|g|^{-1 / 2}\left(|g|^{1 / 2} U_{a}^{b}\right)_{, b} U_{a}^{c}\right) \frac{\partial}{\partial q^{c}}=: B^{c} \partial_{c}
$$

We are going to show that $B^{c}=0$. Using the fact that $|g|_{, \alpha}=0$, we can write

$$
|g|^{-1 / 2}\left(|g|^{1 / 2} U_{a}^{b}\right)_{, b}=|g|^{-1 / 2}\left(|g|^{1 / 2} U_{a}^{A^{\prime}}\right)_{, A^{\prime}}-U_{a, \alpha}^{\alpha},
$$

where $A^{\prime}$ runs over the entire set of indices $a$ and $\alpha$. Then

$$
B^{c}=U_{a}^{\alpha} U_{a, \alpha}^{c}-\left(|g|^{-1 / 2}\left(|g|^{1 / 2} U_{a}^{A^{\prime}}\right)_{, A^{\prime}}-U_{a, \alpha}^{\alpha}\right) U_{a}^{c}=\left(U_{a}^{\alpha} U_{a}^{c}\right)_{, \alpha}-|g|^{-1 / 2}\left(|g|^{1 / 2} U_{a}^{A^{\prime}}\right)_{, A^{\prime}} U_{a}^{c} .
$$

The divergenceless of the vector field $\hat{U}_{a}$ can be reexpressed as

$$
\begin{aligned}
0=|m|^{-1 / 2}\left(|m|^{1 / 2} U_{a}^{A^{\prime}}\right)_{, A^{\prime}} & =|g|^{-1 / 2}|\Omega|^{-1}\left(|g|^{1 / 2}|\Omega| U_{a}^{A^{\prime}}\right)_{,^{\prime}} \\
& =|\Omega|^{-1}|\Omega|_{, A^{\prime}} U_{a}^{A^{\prime}}+|g|^{-1 / 2}\left(|g|^{1 / 2} U_{a}^{A^{\prime}}\right)_{, A^{\prime}},
\end{aligned}
$$

which allows us to rewrite Eq. (7.27) as

$$
B^{c}=\left(U_{a}^{\alpha} U_{a}^{c}\right)_{, \alpha}+|\Omega|^{-1} \hat{U}_{a}(|\Omega|) U_{a}^{c} .
$$

Now, recall that $\hat{U}_{\alpha}=U_{\alpha}^{\beta} \partial_{\beta}, \partial_{\alpha}=\Omega_{\alpha}^{\beta} \hat{U}_{\beta}$. Also

$$
|\Omega|^{-1} \hat{U}_{a}(|\Omega|)=|\Omega|^{-1}|\Omega|\left(-\Omega_{\alpha}^{\beta} \hat{U}_{a}\left(U_{\beta}^{\alpha}\right)\right)=-\Omega_{\alpha}^{\beta} \hat{U}_{a}\left(U_{\beta}^{\alpha}\right) .
$$

Using these results, Eq. (7.29) can be rewritten as

$$
B^{c}=\Omega_{\alpha}^{\beta}\left(\hat{U}_{\beta}\left(U_{a}^{\alpha} U_{a}^{c}\right)-\hat{U}_{a}\left(U_{\beta}^{\alpha}\right) U_{a}^{c}\right)=\Omega_{\alpha}^{\beta}\left(\left(\left(\hat{U}_{\beta}\left(U_{a}^{\alpha}\right)-\hat{U}_{a}\left(U_{\beta}^{\alpha}\right)\right) U_{a}^{c}+U_{a}^{\alpha} \hat{U}_{\beta}\left(U_{a}^{c}\right)\right) .\right.
$$

Recalling that $C_{\beta a}^{\gamma}=-C_{\beta \gamma}^{a}=0$ (compactness and subgroup conditions) we have

$$
\left[\hat{U}_{\beta}, \hat{U}_{a}\right]=C_{\beta a}^{d} \hat{U}_{d}
$$

which implies (using $U_{\beta}^{h}=0$ ) that

$$
\hat{U}_{\beta}\left(U_{a}^{\alpha}\right)-\hat{U}_{a}\left(U_{\beta}^{\alpha}\right)=C_{\beta a}^{d} U_{d}^{\alpha}
$$

and

$$
\hat{U}_{\beta}\left(U_{a}^{c}\right)=C_{\beta a}^{d} U_{d}^{c}
$$

Hence

$$
B^{c}=\Omega_{\alpha}^{\beta} C_{\beta a}^{d}\left(U_{d}^{\alpha} U_{a}^{c}+U_{a}^{\alpha} U_{d}^{c}\right)
$$

which is zero in virtue of the antisymmetry of the structure constants. Therefore 


$$
\left.\Delta_{M}\right|_{\mathscr{W}_{p}}=\Delta_{g}
$$

We would like to emphasize the importance of the compactness of $G$ in the derivation of Eq. (7.36).

\section{EXAMPLE: THE FREE PROPAGATOR ON $S^{2}$ FROM THE FREE PROPAGATOR ON SU(2)}

As a concrete application of the formalism developed in Secs. V and VII we will now consider the motion of a free particle on a sphere $S^{2}$, viewed as the coset space $\mathrm{SU}(2) / \mathrm{U}(1)$. This problem has been studied by Dolan ${ }^{19}$ within the BRST approach. Here we show full equivalence without introducing ghosts.

Making use of the isomorphism $\mathrm{SU}(2) \sim S^{3}$ we may parametrize the group manifold $\mathrm{SU}(2)$ using polar coordinates on $S^{3}$

$$
Q^{A}=(\theta, \phi, \psi), \quad 0 \leqslant \theta \leqslant \pi, \quad 0 \leqslant \phi<2 \pi, \quad 0 \leqslant \psi<4 \pi .
$$

The left-invariant vector fields are

$$
\begin{gathered}
\hat{U}_{1}=\left(\sin \psi \partial_{\theta}-\frac{\cos \psi}{\sin \theta} \partial_{\phi}+\frac{\cos \psi}{\tan \theta} \partial_{\psi}\right) \equiv U_{1}^{B} \partial_{B}, \\
\hat{U}_{2}=\left(\cos \psi \partial_{\theta}+\frac{\sin \psi}{\sin \theta} \partial_{\phi}-\frac{\sin \psi}{\tan \theta} \partial_{\psi}\right) \equiv U_{2}^{B} \partial_{B}, \\
\hat{U}_{3}=\partial_{\psi} \equiv U_{3}{ }^{B} \partial_{B},
\end{gathered}
$$

where $\partial_{B} \equiv \partial / \partial Q^{B}$. They satisfy the $\mathrm{SU}(2)$ algebra

$$
\left[\hat{U}_{A}, \hat{U}_{B}\right]=\epsilon_{A B C} \hat{U}_{C} .
$$

The $\hat{U}_{A}, A=1,2,3$, are Killing vectors for the metric

$$
G^{A B}=U_{C}^{A} U_{D}^{B} \delta^{C D},
$$

which in this coordinate system takes the form

$$
G^{A B}=\left(\begin{array}{ccc}
1 & 0 & 0 \\
0 & \frac{1}{\sin ^{2} \theta} & \frac{-\cos \theta}{\sin ^{2} \theta} \\
0 & \frac{-\cos \theta}{\sin ^{2} \theta} & \frac{1}{\sin ^{2} \theta}
\end{array}\right) .
$$

We will choose the uniparametric subgroup $\mathrm{U}(1)$ generated by $\hat{U}_{3}$ as the gauge group $K$. The simplest choice of the adapted system is then

$$
q^{a}=(\theta, \phi), \quad q^{\alpha}=(\psi) .
$$

In this system, the measure, Eq. (5.14), becomes

$$
\mu(\theta, \phi)=4 \pi \sin \theta
$$

and the propagator, Eq. (5.25), now reads 


$$
{ }_{\mathrm{Ph}}\left\langle\theta^{\prime}, \phi^{\prime}, t^{\prime} \mid \theta, \phi, t\right\rangle_{\mathrm{Ph}}=\frac{1}{16 \pi^{2}} \int_{0}^{4 \pi} d \psi_{2} \int_{0}^{4 \pi} d \psi_{1}\left\langle\theta^{\prime}, \phi^{\prime}, \psi_{2}, t^{\prime} \mid \theta, \phi, \psi_{1}, t\right\rangle
$$

All we have to do now is to substitute for $\left\langle\theta^{\prime}, \phi^{\prime}, \psi_{2}, t^{\prime} \mid \theta, \phi, \psi_{1}, t\right\rangle$ its explicit form, which can be readily found in the literature. Following Schulman ${ }^{20}$

$$
\left\langle\theta^{\prime}, \phi^{\prime}, \psi_{2}, t^{\prime} \mid \theta, \phi, \psi_{1}, t\right\rangle=\frac{1}{16 \pi^{2}} \frac{1}{\sin \Gamma} \sum_{j=0, \frac{1}{2}, 1, \ldots}(2 j+1)(\sin (2 j+1) \Gamma) e^{-(i / 2 I) j(j+1)\left(t^{\prime}-\imath\right)},
$$

where $\Gamma$ is the geodesic distance between two points on $S^{3}$

$$
\cos \Gamma=\cos \left(\frac{\theta}{2}\right) \cos \left(\frac{\theta^{\prime}}{2}\right) \cos \left(\psi_{+}-\psi_{+}^{\prime}\right)+\sin \left(\frac{\theta}{2}\right) \sin \left(\frac{\theta^{\prime}}{2}\right) \cos \left(\psi_{-}-\psi_{-}^{\prime}\right),
$$

with $\psi_{ \pm}=(\psi \pm \phi) / 2$, and $\psi^{\prime}=\psi_{2}, \psi=\psi_{1} . I$ denotes the "moment of inertia" as used in Eq. (4.17) of Rer. 20. We use here notation of Ref. 19. Notice we are taking advantage of the isomorphism between $\mathrm{SU}(2)$ and $S^{3}$. Performing the integrations in Eq. (8.8) we then obtain

$$
\sum_{j=0}^{\infty}(2 j+1) P_{j}(\cos \gamma) e^{-(i / 2 I) \cdot j(j+1)\left(t^{\prime}-t\right)}
$$

where $\gamma$ is the geodesic distance between $q^{\prime}=\left(\theta^{\prime}, \phi^{\prime}\right)$ and $q=(\theta, \phi)$ on $S^{2}$. This is in agreement with Ref. 21, after taking into account the normalizations for our states, given by Eqs. (5.19) and (8.7).

\section{CONCLUSIONS AND OUTLOOK}

In this work we have studied the conditions upon which the first quantize (and then reduce) and the first reduce (and then quantize) approaches are equivalent. There are several lessons that can be drawn from this work, both at a conceptual as well as at a more technical level, keeping in mind that we are considering only systems with Hamiltonian and constraints quadratic and linear in the momenta, respectively. On the one hand, our Lagrangian setting for first class systems reveals quite clearly the ambiguities present in the framework of Dirac's quantization. Within our scheme the one-to-one correspondence between the original Lagrangian and the reduced Hamiltonian quantization is neatly seen. Hence the reduced quantization possesses a certain uniqueness that is lacking in the Dirac approach. This point of view is reinforced when one considers our work with path integrals ${ }^{9}$ where again reduced quantization plays a central role. The discussion in Sec. VI shows that the ambiguities mentioned above make plausible the selection of a specific Dirac quantization which coincides with the reduced first method. Several features of the gauge group relevant to this problem are also mentioned there.

On a more practical level we show rigorously that in the case of coset spaces of compact Lie groups there is a natural selection of Dirac quantization that fully coincides with the reduced quantization. This fact allows us to use results of the quantum theory on the group $G$ to obtain the quantum theory on the coset space $G / K$, with $K$ a subgroup. To make contact with the work of other people we compute the propagator on a two-dimensional sphere $S^{2}=\mathrm{SU}(2) / \mathrm{U}(1)$ by this means.

It would be interesting to extend our approach to the case of constraints quadratic in the momenta-relevant in the study of reparametrization invariant systems like gravity. A close comparison with our work on path integrals should be illuminating. Work on these topics is currently in progress. 


\section{ACKNOWLEDGMENTS}

We are grateful to L. C. Shepley for useful conversations on the geometrical aspects of this article. This work was partly inspired by questions posed to us by J. Polchinski.

C.R.O. would like to express his gratitude to the World Laboratory for its generous support, and to the Guggenheim Foundation for support during the initial stages of this work. J.M.P. would like to thank the Center for Relativity of The University of Texas for its warm hospitality. This research was supported in part by the Robert A. Welch Foundation and NSF Grant No. PHY 9009850 (UT Austin) and NSF Grant No. PHY 8806567 (UT Austin), by the Department of Education Grant No. DE-FG05-87ER40367 (Vanderbilt), by the CICYT of Spain (Project No. AEN-0695), and by a Human Capital and Mobility Grant (ERB4050PL930544).

\section{APPENDIX: THE INVERSE LEGENDRE TRANSFORMATION FOR CONSTRAINED SYSTEMS}

We want to show that from the Hamiltonian (3.4)

$$
H=\frac{1}{2} M^{A B}(Q) P_{A} P_{B}+V
$$

and the primary constraints (3.2)

$$
U_{\alpha}^{A}(Q) P_{A} \approx 0
$$

we can recover the Lagrangian (2.1) which we started with. For this purpose we will perform the inverse Legendre transformation on the Dirac Hamiltonian

$$
H_{D}=\frac{1}{2} M^{A B} P_{A} P_{B}+\lambda^{\alpha} U_{\alpha}^{A} P_{A}+V .
$$

This means we want to find $\left(P_{A}, \lambda^{\alpha}\right)$ in terms of $\left(Q^{A}, \dot{Q}^{A}\right)$, as the solution of the following algebraic system of equations:

$$
\dot{Q}^{A}=\left\{Q^{A}, H_{D}\right\}=M^{A B} P_{B}+\lambda^{\alpha} U_{\alpha}^{A}, \quad U_{\alpha}^{A} P_{A}=0 .
$$

Once this is achieved we will just substitute the momenta as functions of velocity space variables in the expression for the Lagrangian

$$
L=P_{A} \dot{Q}^{A}-H .
$$

Notice that Eq. (A4) involves only half of the Hamilton equations. This is so because this is the one that contains the information about the Legendre map. In virtue of Eq. (A4)

$$
\dot{Q}^{A} U_{\beta A}=\lambda^{\alpha} \Theta_{\alpha \beta},
$$

where

$$
\Theta_{\alpha \beta}=U_{\alpha}^{A} U_{\beta A} \equiv U_{\alpha}^{A} M_{A B} U_{\beta}^{B}
$$

is just the scalar product of the vector fields $\hat{U}_{\alpha}, \hat{U}_{\beta}$ with respect to the metric $M$. Since the vector fields $\hat{U}_{\alpha}, \alpha=1, \ldots, k$ are independent, and the metric $M$ is nonsingular, the matrix $\Theta_{\alpha \beta}$ is invertible. Hence

$$
\lambda^{\alpha}=\Theta^{\alpha \beta} U_{\beta A} \dot{Q}^{A}
$$

and using again Eq. (A4) we obtain 


$$
\dot{Q}^{A}=M^{A B} P_{B}+\Theta^{\alpha \beta} U_{\beta C} \dot{Q}^{C} U_{\alpha}^{A}
$$

from which we get for $P_{B}$

$$
P_{B}=M_{B A} \mathscr{P}_{C}^{A} \dot{Q}^{C}
$$

where

$$
\mathscr{P}_{C}^{A}=\delta_{C}^{A}-\Theta^{\alpha \beta} U_{\beta C} U_{\alpha}^{A}
$$

is the projector in the direction transverse to the orbits. Finally, the Lagrangian becomes

$$
L=\frac{1}{2} M_{C D}^{\perp} \dot{Q}^{C} \dot{Q}^{D}-V
$$

where

$$
M_{C D}^{\perp}=\mathscr{P}_{C}^{A} M_{A B} \mathscr{P}_{D}^{B}
$$

It only remains to show that $M_{C D}^{\perp}=G_{C D}$, where $G_{C D}$ is the metric in the original Lagrangian (2.1). To show this it is convenient to work in the adapted coordinate system, in which the obvious fact that the vectors $\hat{U}_{\alpha}, \alpha=1, \ldots, k$ are null vectors of $M_{C D}^{\perp}$ reveals that it has the form

$$
M^{\perp}=\left(\begin{array}{cc}
M_{a b}^{\perp} & 0 \\
0 & 0
\end{array}\right)
$$

In view of Eq. (4.12), in order to check that $M_{a b}^{\perp}=g_{a b}$ we only need to verify $M^{a A^{\prime}} M_{A^{\prime} b}^{\perp}$ $=\delta_{b}^{a}$. From the definition of $M^{\perp}$ we obtain

$$
M_{A^{\prime} B^{\prime}}^{\perp}=M_{A^{\prime} B^{\prime}}-M_{A^{\prime} \rho} U_{\alpha}^{\rho} \Theta^{\alpha \beta} U_{\beta}^{\sigma} M_{\sigma B^{\prime}}
$$

where $U_{\alpha}^{a}=0$, Eq. (4.9), was used. Finally, recalling that $\delta_{\rho}^{a}=0$ we have

$$
M^{a A^{\prime}} M_{A^{\prime} b}^{\perp}=\delta_{b}^{a}-\delta_{\rho}^{a} U_{\alpha}^{\rho} \Theta{ }^{\alpha \beta} U_{\beta}^{\sigma} M_{\sigma b}=\delta_{b}^{a},
$$

which proves our assertion.

${ }^{1}$ V. Guillemin and S. Sternberg, Symplectic Techniques in Physics (Cambridge, Cambridge, England, 1984).

${ }^{2}$ P. A. M. Dirac, "Generalized Hamiltonian dynamics," Can. J. Math. 2, 129 (1950); Lectures in Quantum Mechanics (Yeshiva University, New York, 1965).

${ }^{3}$ A. Ashtekar and G. T. Horowitz, "On the canonical approach to quantum gravity," Phys. Rev. D 26, 3342 (1982).

${ }^{4}$ K. Kuchar, "Hamiltonian dynamics of gauge systems," Phys. Rev. D 34, 3031 (1986); "Covariant factor ordering of gauge systems," ibid. 34, 3044 (1986).

${ }^{5} \mathrm{P}$. Hájíček and K. Kuchar, "Constraint quantization of parametrized relativistic gauge systems in curved space-times," Phys. Rev. D 41, 1091 (1990).

${ }^{6}$ D. McMullan and J. Paterson, "Covariant factor ordering of gauge systems using ghost variables. I. Constraint rescaling," J. Math. Phys. 30, 477 (1989); "II. States and Observables," ibid. 30, 487 (1989).

${ }^{7}$ E. S. Fradkin and G. A. Vilkovisky, CERN Report TH-2332 (unpublished, 1977); I. A. Batalin and G. A. Vilkovisky, "Relativistic $S$-matrix of dynamical systems with boson and fermion constraints," Phys. Lett. B 69, 309 (1977).

${ }^{8}$ J. D. Romano and R. S. Tate, "Dirac versus reduced space quantization of simple constrained systems," Class. Quantum Gravit. 6, 1487 (1989); K. Schleich, "Is reduced space quantization equivalent to Dirac quantization?," Class. Quantum Gravit. 7, 1529 (1990); R. Loll, "Noncommutativity of constraining and quantizing: A U(1)-gauge model," Phys. Rev. D 41 (1990) 3785; P. Ellicot, G. Kunstatter, and D. J. Toms, "Geometrical derivation of the Faddeev-Popov ansat2," Mod. Phys. Lell. A 4, 2397 (1989); G. Kunstatter, "Dirac versus reduced quantization, a geometric approach," Class. Quantum Gravit. 9, 1469 (1992); M. S. Plyushchay and A. V. Razumov, "Dirac Versus Reduced Phase Space Quantization: Relationship of Two Methods," hep-th/9306017.

${ }^{9}$ C. R. Ordóñe $\angle$ and J. M. Pons, "Equivalence of reduced, Polyakov, Faddeev-Popov, and Faddeev path integral quantization of gauge theories," Phys. Rev. D 45, 3706 (1992). 
${ }^{10} \mathrm{C}$. R. Ordóñez, J. M. Pons, and R. Toldrà, "Unitarity and Batalin-Vilkovisky path integral quantization for gauge theories," Phys. Lett. B 302, 423 (1993).

"C. R. Ordóñez, J. París, J. M. Pons, and R. Toldrà, "Batalin-Vilkovosky analysis for covariant and noncovariant actions," Phys. Rev. D 48, 3818 (1993).

${ }^{12}$ L. D. Faddeev and A. A. Slavnov, Gauge Fields (Addison-Wesley, Reading, MA, 1990).

${ }^{13}$ B. DeWitt, "Quantum theory of gravity. I. The canonical theory," Phys. Rev. 160, 1113 (1967); "The manifest covariant theory," 162, 1195 (1967).

${ }^{14}$ C. Batlle, J. Gomis, J. M. Pons, and N. Roman, "Equivalence between the Lagrangian and Hamiltonian formalism for constrained systems," J. Math. Phys. 27, 2953 (1986); J. M. Pons, "New relations between Hamiltonian and Lagrangian constraints," J. Phys. A 21, 2705 (1988).

${ }^{15}$ B. S. DeWitt, "Dynamical theory of curved spaces. I. A review of the classical and quantum action principles," Rev. Mod. Phys. 29, 377 (1957).

${ }^{16}$ B. S. DeWitt, in General Relativity, An Einstein Centenary Survey, edited by S. W. Hawking and W. Israel (Cambridge, Cambridge, England, 1979).

${ }^{17}$ K. Kuchar, "Consistent factor ordering may be ambiguous," Phys. Rev. D 35, 596 (1987).

${ }^{18} \mathrm{~L}$. O'Raifeartaigh, Group Structure of Gauge Theories (Cambridge, Cambridge, England, 1986).

${ }^{19}$ B. P. Dolan, "BRST symmetry and quantum mechanics of homogeneous spaces," J. Phys. A 23, 4439 (1991).

${ }^{20}$ L. Schulmann, Techniques and Applications of Path Integration (Wiley, New York, 1981).

${ }^{21}$ M. Bohm and G. Junker, "Group Theoretical Approach to Path Integration On Spheres," in Path Summation: Achievements and Goals, Proceedings of the Adriatico Research Conference on Path Integral Methods With Applications, edited by S. Lundvist, A. Ranfagni, V. Sayakanit, and L. S. Schulman (World Scientific, Trieste, 1987). 